\title{
Ergodic Stationary Distribution of Hepatitis C Virus Model Incorporating Two Treatment Effects
}

\author{
Auwal Abdullahi ${ }^{1}$ \\ ${ }^{1}$ Federal University Kashere
}

October 18, 2021

\begin{abstract}
In this paper, the dynamics of Hepatitis $\mathrm{C}$ infectious disease model with two treatment effects are studied through the Ito Stochastic Differential Equations (SDEs). While the first treatment rate reduces the reproduction of virion, the other mitigates the new infections. Though the deterministic behaviour of the model has been extensively studied, little is known about its stochastic properties. Thus, we examine sufficient conditions for the existence and uniqueness of the ergodic stationary distribution of the model via stochastic Lyapunov approach. The existence of a unique positive solution is also studied. The numerical simulations of the SDE model are performed through the Euler-Maruyama method and compared with their deterministic counterparts. The results obtained by SDEs are found to conform to those reported through their deterministic analogues.
\end{abstract}

\section{Hosted file}

Manuscript.pdf available at https://authorea.com/users/441640/articles/542093-ergodicstationary-distribution-of-hepatitis-c-virus-model-incorporating-two-treatment-effects 
figures/FigureOne/FigureOne-eps-converted-to.pdf 
figures/FigureTwo/FigureTwo-eps-converted-to.pdf 
figures/FigureThree/FigureThree-eps-converted-to.pdf 